\title{
Treatment of a Cerebral Thrombophlebitis Associated with a Large Intraparenchymatic Cerebral Hematoma, Resuscitation Department at N'djamena Renaissance Chu
}

\author{
Fall ML*, Ndiaye K, Traore S, Abassi A, Vagba J, Toudjimgar F, Kessel YYC, Berret M
}

UGB 2S

DOI: $10.36347 /$ sjams.2021.v09i02.002

| Received: 22.09.2020 | Accepted: 29.09.2020 | Published: 09.02.2021

*Corresponding author: Dr. Fall Mohamed Lamine

Email: tallafal@yahoo.fr

\section{Abstract}

The association of cerebral thrombophlebitis with cerebral hematoma is an uncommon situation. Hematomas are often satellite or indicative of thrombophlebitis. Its incidence is too low on average $2 \%$ [1], it is increased with the risk factors of hypercoagulability [2]. The diagnosis with clinical polymorphism and imaging thrombophlebitis is often obscured at first glance by the presence of cerebral hematoma. Our observation is a clinical presentation of a large cerebral hematoma indicative of thrombophlebitis in the presence of a convulsive state with laborious therapeutic management. This required a neurosurgical resuscitation team which had targeted thrombophlebitis in its management. The short and long course is often favorable if the management is early, adequate and careful.

Keywords: Treatment - Cerebral Thrombophlebitis - Cerebral Hematoma.

Copyright @ 2021: This is an open-access article distributed under the terms of the Creative Commons Attribution license which permits unrestricted use, distribution, and reproduction in any medium for non-commercial use (NonCommercial, or CC-BY-NC) provided the original author and source are credited.

\section{INTRODUCTION}

Cerebral thrombophlebitis or cerebral venous thrombosis is a rare pathology; its overall incidence is poorly understood and estimated in certain series after autopsy to be less than $2 \%$ of cerebrovascular accidents [1].

The risk is major in the presence of hypercoagulable risk factors with an increased incidence, especially in the peri-partum, and between 19 and $46 \%$ [2]. It quite often affects the young subject with a female predominance $3 \mathrm{~F} / 1 \mathrm{H}[1]$. While cerebral hematoma is pathology quite frequently encountered in neurosurgery, especially in the traumatic context. However, the association of thrombophlebitis and cerebral hematoma is infrequent and often in the presence of risk factors for thrombophlebitis, in this case the satellite or revealing hematomas are small. But the association with a large hematoma is rarely seen. Craniotomy is currently recommended in patients with a cortical hematoma greater than $30 \mathrm{ml}$ and less than 1 $\mathrm{cm}$ from the cortical surface [3].

We pay particular attention to the management of this pathological entity and we illustrate by this management through a clinical presentation.

\section{Patient and Observation}

We report the case of a 37-year-old patient who was admitted to our department for the management of an altered state of consciousness. There was a notion of a traffic accident 4 months before the recent episode. It was collusion between two nonhelmeted motorcyclists who caused their falls. There was no notion of loss of consciousness but there was a right peri orbital swelling with multiple dermabrasions on the head and limbs. He had not yet had a brain CT scan and was concluded to have a mild head injury.

There is no known medical and surgical history in his lifestyle and there is no consumption of alcohol, tobacco, or other psychoaffective substances.

Symptoms re-emerged three days before his hospitalization marked by the onset of sudden onset headaches, intense, diffuse to the whole head; accompanied by vomiting, there was no phosphene or tinnitus. The course was marked by the onset of a generalized tonic-clonic type of convulsive state for which he was treated in our facility. On admission he was obsessed with GCS $=8$ (E2V2M4), isocorous miosis pupils with associated right hemiplegia, and the calves were soft on palpation. Cardiopulmonary auscultation was normal. BMI $=24.9$ almost normal. The clinical parameters were: $\mathrm{BP}=160 / 110 \mathrm{FC}=97 \mathrm{c} /$ 
min, $\mathrm{SpO} 2=87 \%$ in ambient air; $\mathrm{T}=36.5^{\circ} \mathrm{C}$, capillary blood glucose at $1.37 \mathrm{~g} / \mathrm{L}$. An angio-CT scan was performed urgently and revealed a cerebral venous thrombosis of the superior longitudinal sinus, of the left transverse and sigmoid sinus, complicated by a large left temporal hematoma estimated at $40 \mathrm{ml}$ with mass effect (figure 1). The biological assessment shows a CRP at $14.8 \mathrm{mg} / \mathrm{L}$, a hyperleukocytosis at 23,100 elements/ $\mathrm{mm}^{3}$; a platelet count is normal at 294,000 / $\mathrm{mm}^{3}$, a hemoglobin level $=16.8 \mathrm{~g} / \mathrm{dl}$ and no blood crase abnormality. We performed an emergency decompression craniotomy with the realization of a shutter and the installation of a Redon, then hospitalized in the intensive care unit; we used curative anticoagulation after 24 hours of surgery; In view of the persistence of the bleeding in Redon, we readjusted the treatment to the intermediate dose. The postoperative control was favorable in figure 2. The evolution was favorable after a few days of hospitalization in the intensive care unit which required its exeat and in the long term after one year the evolution was still favorable, asymptomatic without deficit.

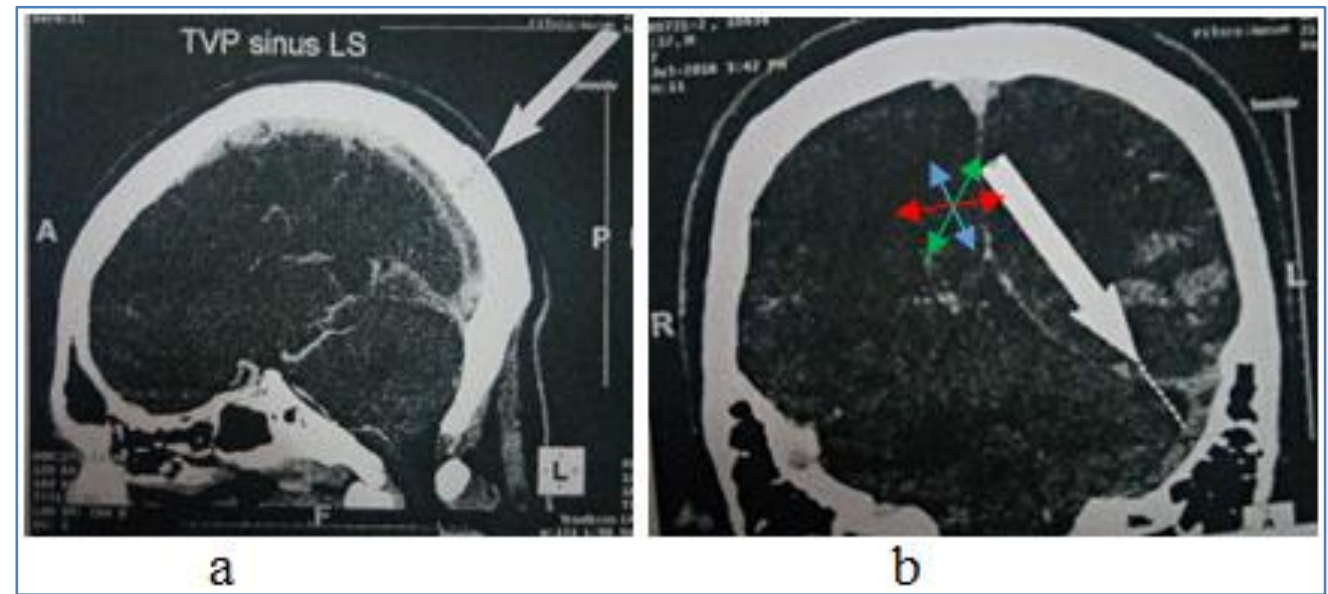

Fig-1: Cerebral thrombosis Superior longitudinal sinus, transverse and left sigmoid sinus a (CVT) and b (hematoma) $\geq 40 \mathrm{ml}$

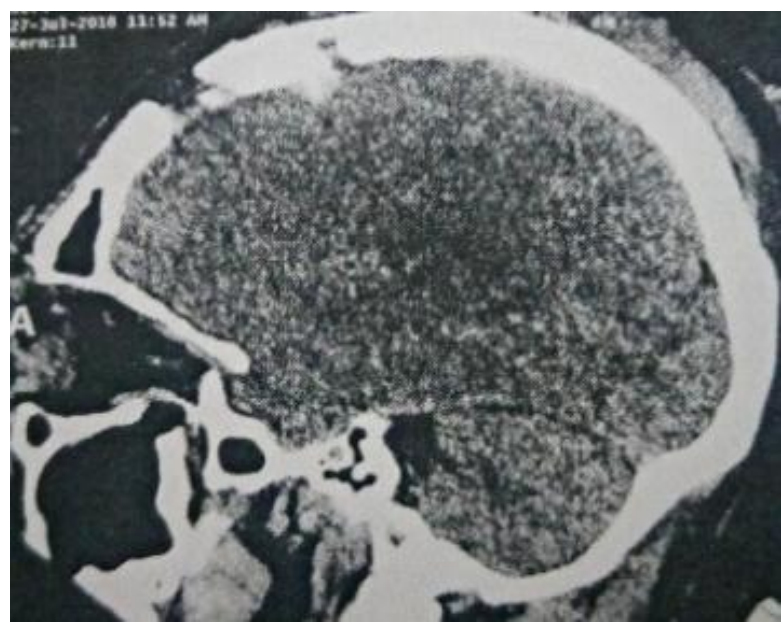

Fig-2: Post-operative evolution

\section{DiscUSSION}

It was the first time that our intensive care unit was confronted with this lesion association and the management was not very laborious at the beginning so it was important for us to share this experience because this pathological entity is rarely encountered in daily practice with any ambiguity. e management of anticoagulants.

Thus after a varied symptomatology precursor of the coma picture; cerebral CT scan revealed cerebral venous thrombosis of the superior longitudinal sinus, the left transverse and sigmoid sinuses, complicated by a large left temporal hematoma, as shown in Figure 1 (a and $\mathrm{b})$.

The volume of a hematoma can be calculated in two ways. The first is the true measurement on the brain scanner after a three-dimensional reconstruction obtained with a spiral scanner. The second method is the calculation by the formula $\mathrm{A} \times \mathrm{B} \times \mathrm{C} / 2$, where $\mathrm{A}$, $\mathrm{B}$ and $\mathrm{C}$ are the diameters of the hematoma in the three directions of space. There is a very good correlation between the two techniques $(r=0.929)$ allowing rapid calculation at bedside (Figure 3) [3]. Craniotomy is currently recommended in patients with a cortical hematoma greater than $30 \mathrm{ml}$ and within $1 \mathrm{~cm}$ of the 
cortical surface. In the context of cerebellar haemorrhages (6-10\% of all haemorrhagic strokes), rapid recourse to surgical evacuation is recommended when there is a change in the state of consciousness or signs of compression of the trunk cerebral [3]. Kaya had reported in his study on post-traumatic intracerebral hematomas, that temporal intracerebral hematomas with a volume $\geq 30 \mathrm{CC}(\mathrm{ml})$ and / or a diameter $\geq 3 \mathrm{~cm}$, are indications for urgent surgery due to the high risk of involvement, they report that according to the recommendations for clinical practice, an intracerebral hematoma or a hemorrhagic contusion of a volume greater than $15 \mathrm{ml}$ with displacement of the midline greater than $5 \mathrm{~mm}$ and obliteration of the basal cisterns should be evacuated as quickly as possible [4]. Toufik had found in his work on cerebral hematomas revealing cerebral venous thrombosis that it is a pathology which affects young adults [5] and Kaaniche had reported the same observation in a 33-year-old patient [6]. Abarca had reported two cases of cerebral hemorrhage secondary to developmental venous anomaly thrombosis in two female and male patients aged 28 and 38 , respectively [7]. One of which had presented in the same clinical context as our patient, i.e. in a table of tonic-clonic convulsive seizures Of which on CT, MRI and magnetic resonance angiography (MRA), had objectified a vein thrombosis adjacent to the hematoma consistent with a partially thrombosis vessel. Digital subtraction angiography confirmed the presence of a filling defect in the draining vein of a developmental venous anomaly (DVA) characteristic of the model [7].

The work of $\mathrm{Oz}$ had confronted a young patient operated on for pathology of the meniscus under spinal anesthesia and who complained of intense postoperative headaches requiring the performance of a cerebral CT scan which had demonstrated a subarachnoid hemorrhage with thrombosis of the venous sinus. They stated that this is a first situation encountered and that the evolution has been favorable with heparin [8]. Joulali had reported a deceptive case of subarachnoid hemorrhage and parenchymal hematoma indicative of cerebral venous thrombosis in a 37-year-old patient on estrogen-progestogen-based oral contraception in front of consciousness disorders on the table of subacute headaches and photophobia with a hemiplegia. In whom the initial scan shows subarachnoid hemorrhage. A control cerebral CT scan carried out before the secondary onset of tonic-clonic convulsive seizures followed by a post-critical coma, demonstrated the appearance of a frontal hematoma associated with peri-lesional edema. The cerebral arteriography carried out as part of the etiological assessment and with a therapeutic objective of subarachnoid hemorrhage had suspected cerebral thrombophlebitis then the complement of cerebral angio-CT found a thrombosis of the anterior two thirds of the upper longitudinal sinus with a frontal infarction right, an intra-parenchymal hematoma and subarachnoid hemorrhage [5]. That said, the demonstration of the lesion association is sometimes laborious but sometimes with successful management; the lesions observed are almost similar to those found in our observation. Yuan-Pi Ko et al. showed that the diagnosis of a cerebral thrombophlebitis and hematoma association was not always obvious, the acute subarachnoid hematoma has often been reported as the initial sign of cerebral venous thrombosis and the diagnosis is a real challenge because the symptomatology is often non-specific [2].

Taoufik had shown that the clinical picture is very varied and often misleading; the five most frequent signs are headaches in more than $80 \%$ of cases, focal deficits, generalized or non-generalized convulsions, papillary edema and pain disorders consciousness ranging from confusion to deep coma [5]. As for our patient, it was a presentation of the convulsions in the form of status epileptics.

Saposnik in his study on the diagnosis and management of cerebral venous thrombosis showed that cerebral venous thrombosis is a rare form of stroke, usually affecting young people. Despite advances in diagnostics in recent years, diagnosis and management can be difficult due to the diversity of underlying risk factors and the lack of a consistent treatment approach [9]. Several factors have been associated with cerebral venous thrombosis, only some are reversible. Previous medical conditions (thrombophilia, inflammatory bowel disease), transient situations (pregnancy, dehydration and infection), certain medications (e.g., oral contraceptives, drug addiction) and unforeseeable events (e.g. head trauma) are predisposing conditions [9].

Kaaniche $\mathrm{F}$ et al. had found cerebral venous thrombosis, in particular of the left lateral sinus without associated subarachnoid hemorrhage, which occurred in a 32-year-old girl two weeks after a benign head trauma. She had presented herself with a psychiatric chart of amnesia of the facts. All cause of thrombophilia was eliminated, only the traumatic cause was found [6]. And it has been shown that brain tissue is rich in thromboplastin, which a powerful activator of coagulation is, and that it is activated by the cascade of reactions in an injured brain [10].

As for our patient, the only contributing factor was head trauma, reported by several authors including Saposnik and Astrup [9, 10]. Bonneville in her study on imaging of cerebral venous thromboses found hemorrhage due to venous stasis and rupture of the blood-brain barrier in $30 \%$ of cases [11].

Our initial therapeutic management consisted of airway management by controlled ventilation under sedation with fentanyl and Hypnovel with adapted resuscitation measures followed by decompressive craniotomy by evacuation of the intraparenchymal 
hematoma with the initial setting of anticoagulation to LMWH-based curative dose for cerebral thrombophlebitis. We were faced with a significant production of very haematic cerebral Redon after 24 hours, which allowed us to readjust the dose by reducing to an iso-coagulant dose according to the patient's weight. The short and long term outcome was favorable with complete recovery of the patient, released without significant neurological sequelae. Abarca had proceeded in the same way as us with the primary realization of an urgent craniotomy and a partial evacuation of the hematoma then a systemic anticoagulation which he had started and four days after the surgery, the sedation was stopped and the evolution was favorable in his patients [7]. Joulali had put his patient on Nimodipine by injection with resuscitation measures. The patient was intubated ventilated and sedated with Hypnovel and fentanyl, followed by antiepileptic therapy with sodium valproate and clonazepam. In their case, anticoagulation was started as soon as the CVT based on low molecular weight heparin was confirmed subcutaneously linked by antivitamin $\mathrm{K}$ without significant bleeding complications [5]. Oppenhein reported that the treatment is based on symptomatic, etiological and above all anti-thrombotic management even in the presence of subarachnoid hemorrhage or parenchymal hematoma [12].

\section{CONCLUSION}

The association of cerebral thrombophlebitis with cerebral hematoma is a rare disease entity. Its incidence is not well known but some estimated it in series of autopsies to less than $2 \%$ of the cerebrovascular accidents. It quite often affects the young subject with a female predominance $3 \mathrm{~F} / 1 \mathrm{H}$. The diagnosis is often made in front of a cerebral hematoma in an often non-traumatic context and its management is laborious. It requires an experienced neuro-resuscitation team and it is essentially based on the management of anticoagulants, preferably LMWHs for the dissolution of thrombophlebitis after control of the cerebral hematoma. The outcome is often favorable if the treatment is early and adequate.

\section{REFERENCES}

1. Midaoui E, Souirti Z, Messouak O, Belahsen MF. Thrombose veineuse cérébrale. AMETHER. Oct 2009; 1(1): 4450.
2. Yuan-Pi Ko, Chin-Yuan Hsu, Chih-Lin Yang, ShoJen Cheng at all. Postcesarean Subarachnoid Hemorrhage as the Initial Presentation of Cerebral Venous Thrombosis. Taiwan J Obstet Gynecol. Sept. 2007; 46(3)

3. Velly L, Bruder N, Pellegrini L. hématomes intracrâniens non traumatiques. Neurochirurgie. 2012. 20: 9.

4. Kaya JM, Alliez JR, Diallo O, Balan C, Alliez B. Les Hématomes Intracérébraux Post-Traumatiques. AJNS 2007; 26(1).

5. Joulali T, Derkaoui A, Besri S, Malki M. Hémorragie méningée et hématome parenchymateux révélant une thrombose veineuse cérébrale. Pan African Medical Journal. 2015; 20: 293

6. Kaaniche F, Chaari O, Turki O, Chelly H, Bouaziz M. Thrombophlébite cérébrale révélée par des troubles psychiatriques. Journal des maladies vasculaires. 2015; 40: 192-95

7. J Abarca-Olivas, C Botella-Asunción, LA Concepción-Aramendía, JJ Cortés-Vela, JI Gallego-León, F Ballenilla-Marco. Deux cas d'hémorragie cérébrale secondaire à une thrombose d'anomalie veineuse développementale. Neurocirugia (Astur) Juin. 2009; 20 (3): 265-71.

8. $\mathrm{Oz} \mathrm{O}$, Akgun H, Yücel M, Battal B. Cerebral venous thrombosis presenting with subarachnoid hemorrhage after spinal anesthesia. Acta Neurol Belg. 2011 Sep;111(3): 237-40.

9. Saposnik G. Diagnosis and Management of Cerebral Venous Thrombosis A Statement for Healthcare Professionals from the American Heart. Association/American Stroke Association. Stroke April. 2011; 1158-1192

10. Astrup T. Assay and content of tissue thromboplastin in different organs. Thromb death haemorrh. 1965; 14: 401- 16.

11. Bonneville F. Imagerie des thromboses veineuses cérébrales. Journal de Radiologie Diagnostique et Interventionnelle. Decemb. 2014: 95(12): 1130-35

12. Oppenheim C, Domigo V, Gauvrit JY, Lamy C, and al. Subarachnoid Hemorrhage as the Initial Presentation of Dural Sinus Thrombosis. AJNR Am J Neuroradiol. March. 2005; 26: 614-17. 